\title{
The Activation of MEK1 by Enhanced Homodimerization Drives Tumorigenesis
}

Jimin Yuan ${ }^{1}$, Wan Hwa Ng${ }^{1}$, Zizi Tian ${ }^{3}$, Jiajun Yap ${ }^{1}$, Manuela Baccarini ${ }^{4}$, Zhongzhou Chen ${ }^{3}$ and Jiancheng $\mathrm{Hu}^{1,2^{*}}$

'Division of Cellular and Molecular Research, National Cancer Centre Singapore; 11 Hospital Drive, 169610, Singapore.

${ }^{2}$ Cancer and Stem Cell Program, Duke-NUS Medical School; 8 College Road, 169857, Singapore. ${ }^{3}$ State Key Laboratory of Agrobiotechnology, College of Biological Sciences, China Agricultural University, Beijing 100193, China

${ }^{4}$ Max F. Perutz Laboratories, University of Vienna, Doktor-Bohr-Gasse 9, 1030 Vienna, Austria.

\section{Running Title: The MEK1 mutants with elevated dimer affinity in cancer genomes}

Keywords: RAF/MEK/ERK kinase cascade, MEK, oncogenic mutations, dimerization, allosteric activity, drug resistance, cancer

Corresponding Author:

Dr. Jiancheng $\mathrm{Hu}$

Division of Cellular and Molecular Research, National Cancer Centre Singapore;

11 Hospital Drive, 169610, Singapore;

Tel: 65+62237241, E-mail: hu.jiancheng@nccs.com.sg 


\section{Summary}

Hyperactive RAS/RAF/MEK/ERK signaling has a well-defined role in cancer biology. Aberrant pathway activation occurs mostly upstream of MEK; however, MEK mutations are prevalent in some cancer subsets. Here we show that cancer-related MEK mutants can be classified as those activated by relieving the inhibitory role of helix $A$, and those with in-frame deletions of $\beta 3-\alpha C$ loop, which exhibit differential resistance to MEK inhibitors in vitro and in vivo. The $\beta 3-\alpha C$ loop deletions activate MEK1 through enhancing homodimerization that can drive intradimer cross-phosphorylation of activation loop. Further, we demonstrate that MEK1 dimerization is required both for its activation by RAF and for its catalytic activity towards ERK. Our study identifies a novel group of MEK mutants, illustrates some key steps in RAF/MEK/ERK activation, and has important implications for the design of therapies targeting hyperactive RAS/RAF/MEK/ERK signaling in cancers.

\section{Introduction}

The RAF/MEK/ERK kinase cascade plays a central role in cell biology, and its hyperactivation results in many human diseases, especially cancers(1-3). Genetic alterations that aberrantly activate this kinase cascade in cancers mainly occur in receptor tyrosine kinases (RTKs), RAS, and RAF, while oncogenic mutations of MEK and ERK are rare. However, recent genomic sequencings showed that MEK mutations are highly prevalent in some specific type of cancers such as IGHV4-34+ hairy cell leukemia and non-BRAF(V600E) Langerhans cell histiocytosis(4-7). To characterize oncogenic MEK mutations and explore how these mutations turn on MEK has important implications for the development of therapeutic inhibitors and for the treatment of MEK-driven cancers.

As a core component of the RAF/MEK/ERK kinase cascade, MEK is a dual specific kinase that transmits a signal from active RAF to phosphorylate $\operatorname{ERK}(8,9)$. The regulation of MEK is complex and not yet completely understood. In RAF-driven cancers, active RAF mutants, such as BRAF(V600E), activate MEK by phosphorylating its activation segment, which results in a high level of phosphoMEK(10). In contrast, the level of phospho-MEK is much lower in RTK/RAS-driven cancers, although they have similar levels of MEK activity as indicated by comparable phospho-ERK level $(11,12)$. This suggests that wild-type active RAF activates MEK in a different way than BRAF(V600E). In addition, some studies have indicated that AL-autophosphorylation of MEK might also contribute to its activation(13-15), adding another layer of complexity to the regulation of MEK activity.

The activity of MEK is regulated not only by the AL-phosphorylation/dephosphorylation but also by its interactions with other components of RAF/MEK/ERK kinase cascade(16-18). The heterodimerization of RAF/MEK facilitates MEK activation(19), while the MEK/ERK interaction contributes ERK phosphorylation(20). Moreover, MEK has been shown to form face-to-face homodimers in which the activation loop of one protomer aligns with the catalytic site of the other(21), suggesting a potential intra-dimer transphosphorylation. In addition, MEK also forms heterodimers between its two isoforms, which regulates the duration of ERK signaling(22). However, whether and how MEK interactome alterations contribute to hyperactive ERK signaling-driven tumorigenesis remains unkown. 
In this study, we have conducted a survey of cancer genomic databases, which leads to the identification of a novel group of cancer-related constitutively active MEK1 mutants with in-frame deletions in the $\beta 3-\alpha C$ loop. These MEK1 mutants exhibit a strong oncogenic potential, but differential sensitivity to MEK inhibitors in clinic therapy or trials. We find that $\beta 3-\alpha C$ loop deletions enhance the homodimerization of MEK1, which drives an intra-dimer transphosphorylation of activation loops between two protomers. Further, we investigated the role of MEK dimerization on the signaltransmission among RAF/MEK/ERK kinase cascade by using these MEK1 mutants with enhanced but differential dimer affinity. Our data show that MEK1 is phosphorylated by active RAF in a dimerdependent manner and functions as a dimer to phosphorylate ERK1/2, indicating that the dimerization of MEK is critical for its function. Together, this study not only discloses a novel group of oncogenic MEK1 mutants but also uncovers the regulatory mechanism of MEK1 in the RAF/MEK/ERK kinase cascade, and have prominent significance in both drug design and clinic therapy against hyperactive RAS/RAF/MEK/ERK signaling-driven cancers.

\section{Results}

The MEK mutation spectrum in cancer genomes.

116 To survey and characterize cancer-related MEK mutants, here we interrogated the ICGC

117 (International Cancer Genome Consortium) database, the cBioportal for Cancer Genomics database,

118 and the COSMIC (Catalogue of Somatic Mutations in Cancer) database. Together with those reported

119 in literatures, all MEK mutants in cancer genomes were listed in supplementary table 1, and the MEK

120 mutation spectrum was constructed as in Figure1A and supplementary Figure 1A. According to our

121 statistic analysis, MEK1 is the dominant isoform of oncogenic alterations, and has four hot spots,

122 which include the negative regulatory helix $A$ (residue 44 61) (I), the $\alpha C$ - $\beta 4$ loop (II), the $\beta 7$ - $\beta 8$ loop

123 (III), and the $\beta 3-\alpha C$ loop (IV) (Fig1B-C). It has been shown that the negative regulatory helix $A$

124 interacts with the kinase domain and stabilizes its inactive conformation, whose disruption will trigger

125 the kinase activity of MEK1(23). According to their structural distributions, we thought that MEK1

126 alterations/mutations in region I, II, and III activate MEK1 likely through relieving the inhibition of helix

127 A since altered residues are involved in the interaction of helix A with the kinase domain. In contrast,

128 the alterations of $\beta 3-\alpha C$ loop that consist of variable in-frame deletions represent for a novel group of

129 MEK1 mutations, which activate MEK1 via a distinct mechanism.

131 MEK1 mutants with in-frame deletions of $\beta 3-\alpha C$ loop are oncogenic and have differential 132 resistance to MEK inhibitors.

133 To characterize MEK1 mutants with in-frame deletions of $\beta 3-\alpha C$ loop, we measured their activity in vivo and in vitro. As shown in Figure 1D-E and supplementary Figure 1B-D, these mutants activate ERK1/2 when expressed in 293T cells or immortalized fibroblasts with or without RAFs, or upon purification from 293T transfectants, suggesting that they have constitutive activity independent of upstream stimuli. Further, all MEK1 mutants except MEK1 $(\Delta \mathrm{HLEl})$ that has the lowest activity exhibit a strong oncogenic potential, which transform cells independently of RAFs when expressed in immortalized fibroblasts (Fig $1 \mathrm{~F}$ and S1E-F) and induce fibroblastomas when these transformed 
140 fibroblasts were xenografted into NOD-SCID mice (data shown below). Together, these data indicated

141 that MEK1 mutants with in-frame deletions of $\beta 3-\alpha C$ loop could function as cancer drivers.

142 We next determined whether MEK inhibitors applied in therapy or in clinic trials(24) could be used

143 to target oncogenic MEK1 mutants with variable deletions of $\beta 3-\alpha C$ loop. By using 293T transfectants

144 and stable fibroblast lines expressing these MEK1 mutants, we found that, among all inhibitors that

145 tested in this study, Tramentinib was the most effective in blocking the activity of MEK $1(\Delta \mathrm{EI})$, while

146 MEK1 $(\triangle \mathrm{IHLEIK}$ ) was resistant to all inhibitors (Fig 2A and S2A-C). To further evaluate the ability of

147 Tramentinib to target MEK1 mutants with $\beta 3-\alpha C$ loop deletions in vivo, we here constructed

148 xenografted melanomas by using MeWo melanoma cell lines that stably express these MEK1

149 mutants. Together with xenografted fibroblastomas above, we found that all tumors harboring

$150 \mathrm{MEK} 1(\triangle \mathrm{IHLEIK})$ had a robust resistance to Tramentinib treatment in contrast to those harboring

151 MEK1 $(\Delta \mathrm{EI})$ or MEK1(DD) (Fig 2B-D and S2D-G).

152

153

\section{In-frame deletions of $\beta 3-\alpha C$ loop activate MEK1 through enhancing homodimerization.}

154

In-frame deletions of $\beta 3-\alpha C$ loop define a new group of oncogenic MEK1 mutations. However, how these alterations trigger the kinase activity of MEK1 remains unknown. Recently, we and other groups found that variable $\beta 3-\alpha C$ loop deletions could activate RAF kinases through promoting homodimerization $(25,26$ ) (Yuan and $\mathrm{Hu}$, unpublished data). Here we assumed that these MEK1 mutants are activated via a similar manner though it has been shown that, different from the side-toside dimer of RAF kinases, MEK1 forms a face-to-face dimer(21). To testify this notion, we firstly measured the dimer affinity of MEK1 mutants with variable $\beta 3-\alpha C$ loop deletions by using PAGE with low SDS $(0.1 \%)$ and microscale thermophoresis (MST) methods. As shown in Figure $3 A$ and S3A, MEK1 mutants with in-frame $\beta 3-\alpha C$ loop deletions have elevated dimer stability/affinity with $\operatorname{MEK} 1(\Delta \mathrm{IHLEIK})>\operatorname{MEK} 1(\Delta \mathrm{EI})>\operatorname{MEK} 1(\mathrm{DD})>\operatorname{MEK} 1(\triangle \mathrm{HLEI})>\mathrm{WT}$. A compound mutation that includes N78G, V224G, F311A, and L314A (called as GGAA below) in the dimer interface $(21,22)$, dramatically decreased both the dimer stability/affinity and the activity of MEK1 mutants with $\beta 3-\alpha C$ loop deletions (Fig 3B and S3B), which further supports our hypothesis.

The activity of MEK1 is triggered by the enhanced homodimerization, suggesting that it has both allosteric and catalytic functions as RAF kinases do. To examine the allosteric activity of MEK1 by protein kinase co-activation assay(27-33), we created kinase-dead allosteric activators by deleting the negative regulatory helix $\mathrm{A}$ ( $\triangle \mathrm{E} 44-\mathrm{E} 51)$ and fusing the catalytic spine (V82F mutation) in MEK1 mutants with variable deletions of $\beta 3-\alpha C$ loop (Fig S3C). MEK1 $(\Delta E \mathrm{El} /$ helix $A-/ V 82 F)$ was the only mutant capable of activating wild-type MEK1 when coexpressed in 293T cells, and its allosteric activity was blocked by the GGAA mutation in dimer interface (Fig S3D-E). The subtle allosteric activity of MEK1 $\left(\triangle \mathrm{HLEI} /\right.$ helix $\left.\mathrm{A}^{-} / \mathrm{V} 82 \mathrm{~F}\right)$ and MEK1 $\left(\triangle \mathrm{IHLEIK/} \mathrm{helix} \mathrm{A}^{-} / \mathrm{V} 82 \mathrm{~F}\right)$ might arise from their weak ability to dimerize with receiver (Figure S3F). Further, we examined whether a compound MEK1 mutant mimicking active MEK1 with AL-phosphorylation has allosteric activity. As shown in Figure 3C, the MEK1 mutant containing the helix A-, the V82F, and the DD (S218D, S221D), could 
179 293T cells. Together, these data demonstrate that wild-type MEK1 has an ability of allosteric activator 180 once activated.

181 The AL-phosphorylation is an indicator of MEK fully activation(8). To explore the molecular basis

182 of MEK1 activation by enhanced homodimerization, we checked the AL-phosphorylation status of

183 MEK1 mutants with variable deletions of $\beta 3-\alpha C$ loop, and found that the AL was phosphorylated in

$184 \operatorname{MEK} 1(\Delta \mathrm{EI})$ and MEK1 $(\Delta \mathrm{HLEI})$ but not in MEK1( $\Delta \mathrm{IHLEIK})$. Moreover, their AL-phosphorylation was

185 blocked by either catalytic spine fusion or dimer interface disruption (Fig 3D and S3G). This data

186 suggested that MEK1 was turned on by homodimerization and then auto-phosphorylated its AL, and

187 that MEK1 $(\triangle \mathrm{IHLEIK})$ might mimic an intermediate locked in a transitional status. To further determine

188 whether the AL-autophosphorylation occurs in cis or trans, we purified the receiver from MEK1 co-

189 activation transfectants and found that its AL was barely phosphorylated (Fig 3E), suggesting that the

190 homodimerization drives a intradimer cross-phosphorylation of two protomers. This finding was also

191 supported by that the non-phosphorylatable AL-mutation (S217A/S221A) reduces the activity of

$192 \operatorname{MEK} 1(\Delta \mathrm{EI})$ and MEK1 $(\Delta \mathrm{HLEI})$ by $60 \sim 70 \%$ but not that of MEK1( $\triangle \mathrm{IHLEIK})$ (Fig $3 \mathrm{~F}$ ). Overall, our data

193 demonstrated that in-frame deletions of $\beta 3-\alpha C$ loop activate MEK1 via homodimerization-driven cross-

194 phosphorylation (Fig 3G).

195

MEK1 transduces a signal from active RAF to activate ERK in a dimer-dependent manner.

197

The interactions among different components play a crucial role in the signal transmission of RAF/MEK/ERK kinase cascade(16-18). Recently, we demonstrated that active RAF kinases function as a dimer to phosphorylate MEK and the MEK-binding ability of both RAF protomers is required for this process (Yuan and $\mathrm{Hu}$, unpublished). To further understand the molecular basis of this process, here we determined whether MEK is phosphorylated by active RAF as a dimer. Thus, we co-

202 expressed BRAF(V600E) or K-RAS(G12V) with wild-type MEK1, or mutants with impaired/enhanced dimer, and found that both BRAF(V600E) and K-RAS(G12V) induced the AL-phosphorylation of wildtype MEK1 and dimeric MEK1( $\triangle \mathrm{IHLEIK}$ ) but not of dimer-impaired MEK1(GGAA) mutant, though the level of AL-phosphorylation differed (Fig 4A). This suggests that MEK dimerization is indispensable for its AL-phosphorylation by active RAF. Since the activation of MEK requires its heterodimerization with RAF, and the heterodimer interface on MEK mostly overlaps with its homodimer interface(19), it is possible that the impaired AL-phosphorylation of MEK1(GGAA) induced by BRAF(V600E) or KRAS(G12V) results from a loss of heterodimerization with RAF. To investigate this possibility, we examined the interaction of MEK1 mutants with $\operatorname{BRAF}(\mathrm{V} 600 \mathrm{E})$ by co-immunoprecipitation, and found that both MEK1(GGAA) and MEK1 $(\triangle \mathrm{IHLEIK})$ showed a reduced complex formation with BRAF(V600E) compared to wild-type MEK1 (Fig 4B), indicating that the impaired ALphosphorylation of MEK1(GGAA) is not related to loss of heterodimerization with RAF. In addition, the waeker AL-phosphorylation of MEK1 ( $\triangle \mathrm{IHLEIK})$ induced by K-RAS(G12V) but not by BRAF(V600E) compared to wild-type MEK1 suggested that the heterodimerization of MEK1 with RAF is important for its activation by wild-type RAF. Together, these data indicate that the dimerization of MEK1 is required for its phosphorylation by active RAF. 
We next investigated whether active MEK functions as a dimer and requires dimeric ERK as substrate for phosphorylation as active RAF does. Indeed, the disruption of dimer interface by GGAA mutation impairs the activity of MEK1(DD) toward ERK which can be restored by GST fusion

221 (Fig 4C), suggesting that active MEK really acts as a dimer. In contrast, wild-type and monomeric

222 ERK were phosphorylated equally when co-expressed with BRAF(V600E) or MEK1(DD) in 293T cells

223 (Fig 4D), indicating that the dimerization of ERK is dispensable for its activation by MEK.

224

\section{Discussion}

226

In this study, we systematically characterized cancer-related MEK mutations, and found that MEK mutants could be classified as two groups: (1) mutants in which the inhibitory interaction between helix $A$ and kinase domain is relieved, and have been shown sensitive to MEK inhibitors (5, 6, 34, 35); (2) mutants with in-frame deletions of $\beta 3-\alpha C$ loop, which are turned on through enhanced homodimerization and have differential resistance to MEK inhibitors. This finding provides a guideline for the targeted therapy of MEK-driven cancers.

MEK1 mutants with variable deletions of $\beta 3-\alpha C$ loop exhibit enhanced but variable dimer affinity, different degrees of AL-phosphorylation, and differential drug resistance. Previous studies have shown that, although the activity of ERK in both BRAF(V600E)- and Ras mutant-driven cancers is comparable, the AL-phosphorylation of MEK and the sensitivity to MEK inhibitors are quite different(10-12), raising the possibility that the inhibitor sensitivity correlates with the ALphosphorylation status of MEK. Our study excludes this possibility in MEK1 mutants with variable deletions of $\beta 3-\alpha C$ loop (data not shown), but whether their different inhibitor sensitivity results from their differential dimer affinity requires further study.

The activation of the RAF/MEK/ERK kinase cascade is a complex process involving physical interactions among its components(16-18). This process is initiated by RAF dimerization, which not only turns on RAF but also facilitates the activation of MEK. Then MEK could be activated by RAFmediated AL-phosphorylation, but also by homodimerization-driven AL-auto-phosphorylation. Like RAF kinases, the dimerization of MEK is required for both its activation and catalytic function. Together with previous findings, we propose the following model for the activation of the

247 RAF/MEK/ERK kinase cascade (Fig 4E): (1) In quiescent cells, RAFs form face-to-face heterodimers 248 with MEKs. (2) Upon stimulation, active RAS (also dimers) recruit RAF/MEK heterodimers to the 249 plasma membrane through the RBD domain of RAFs. There, RAF/MEK heterodimers form transient tetramers by the back-to-back dimerization of RAFs. (3) The back-to-back dimerization of RAFs activates RAFs by inducing cis-autophosphorylation of the AL, and on the other hand, loosens RAF/MEK heterodimers and facilitates the assembly of MEK homodimers on their surface. (4) Active RAF dimers phosphorylate one or both protomers of MEK dimers docking on their surface, and the phospho-MEK protomers can also cross-phosphorylate the other protomers in the context of MEK homodimers. (5) Once both protomers are phosphorylated, MEK dimers can be released from the RAF dimers and phosphorylate ERKs. Semi/non-phosphorylated active MEK dimers docking on the surface of active RAF dimers can also directly phosphorylate ERKs. Constitutively active RAF 
mutants including BRAF(V600E) also activate MEK in a dimer-to-dimer manner. According to this model, allosteric inhibitors that disrupt RAF-MEK and MEK-MEK dimerization would have a high efficacy to block hyperactive ERK signaling in cancers harboring genetic alterations in MEK or upstream of MEK, and be ideal next-generation drugs for cancer therapy.

262

\section{Materials and Methods}

Antibodies, Biochemicals, Cell lines, and Plasmids

Antibodies used in this study include: anti-phosphoERK1/2, anti-phosphoMEK1/2, and antiMEK1/2 (Cell Signaling Technology); anti-FLAG (Sigma); anti-HA (Novus Biologicals); anti-ERK1/2 (AB clonal); anti-Ki67 (Abcam); and HRP-labeled secondary antibodies (Jackson Laboratories). Trametinib, GDC0623, Cobimetinib, AZD6244, and Binimentinib were purchased from Medchemexpress. All other chemicals were obtained from Sigma. Wild-type, BRAF-- and $\mathrm{CRAF}^{-/-}$ fibroblasts were generated in previous study(36). MeWo melanoma cell line was obtained from ATCC. Plasmids encoding RAS, RAF, MEK, ERK, and their mutants were constructed by Gibson assembly. pCDNA3.1(+) vector (Invitrogen) was used for transient expression; retro- or lenti-viral vectors (Clontech) for stable expression; and pET-28a (Novagen) for bacterial expression.

Protein expression and Purification

6xhis-tagged ERK2(K52A) was expressed in BL21(DE3) strains and purified by using Nickel column (Qiagen) as described before(37). FLAG-tagged MEK1, MEK1( $\triangle E \mathrm{EI}), \mathrm{MEK} 1(\Delta \mathrm{IHLEIK})$ were expressed in 293T cells and purified by using anti-FLAG affinity gel and 3XFLAG peptide (Sigma) and following manufacturer's protocol.

Cell Culture, Transfection, and Transduction

All cell lines were maintained in DMEM medium with 10\% FBS (Hyclone). Cell transfection were carried out by using the Biotool transfection reagent. To generate stable cell lines that express MEK1 mutants, viruses were prepared and applied to infect target cells according to our previous studies 37 , 38). Infected cells were selected by using antibiotics.

Immunoprecipitation, In Vitro Kinase Assay, and Western Blotting

Immunoprecipitations were performed as described previously(27-29). Briefly, whole-cell lysates were mixed with either anti-HA,or anti-FLAG beads (Sigma), rotated in cold room for $60 \mathrm{~min}$, and washed three times with RIPA buffer. For in vitro kinase assays, the immunoprecipitants were washed once with kinase reaction buffer (25 mM HEPES, $10 \mathrm{mM} \mathrm{MgCl2,} 0.5 \mathrm{mM} \mathrm{Na3VO} 4,0.5 \mathrm{mM}$ DTT, pH 7.4), then incubated with $20 \mu \mathrm{l}$ kinase reaction mixture (2 ug substrate and $100 \mathrm{mM}$ ATP in $20 \mu \mathrm{l}$ kinase reaction buffer) per sample at room temperature for $10 \mathrm{~min}$. Kinase reaction was stopped by adding 
Immortalized MEFs infected with retroviruses encoding target proteins were plated at $5 \times 10^{3}$ cells per $60 \mathrm{~mm}$ dish, and fed every other day. 12 days later, cells were fixed with $2 \%$ formaldehyde and stained with Giemsa solution (Sigma).

Animal studies

For xenograft experiments, female NOD/SCID mice (6 8 weeks) were subcutaneously injected with $3 \times 10^{6}$ cells per mice in $1: 1$ matrigel (Corning). Tumor volumes were monitored by digital calipers twice a week and calculated using the formula: volume $=(\text { width })^{2} \times$ length/2. Tramentinib was administered orally $(2 \mathrm{mg} / \mathrm{kg}$ ) every other day when tumors reached an average volume of $\sim 50-60$ $\mathrm{mm}^{3}$. At the experiment endpoint, mice were euthanized and tumors were harvested for ex vivo analysis.

Immunohistochemistry staining

Tumors were fixed in $10 \%$ buffered formalin overnight and embedded according to standard procedures. Tumor sections were cut to 4 um thickness, mounted on glass slides, and air-dried at room temperature. After antigen retrieval, tumor sections were stained with antibodies and then with hematoxylin. Images of tumor sections were taken with a bright light microscope at X10.

\section{Author Contributions}

J.Y. and J.H. designed the study; J.Y. and J.H. searched databases/literatures for MEK mutations in cancer genomes; M.B. prepared RAF-knockout cell lines; J.Y., W.H.N, Z.T., J.J.Y., and J.H. carried out molecular biology, biochemistry, and cell biology experiments; J.Y. constructed mouse xenograft models and performed immunohistology analysis; M.B., Z.C. and J.H. supervised all experiments and interpreted experimental data; J.H. wrote the manuscript; M.B. revised manuscript; and all authors commented and approved the manuscript.

\section{Acknowledgement}

We thank Dr. Hui Kam Man, Dr. Kanaga Sabapathy, Dr. Paula Lam, Dr. David Virshup, Dr. Wang Mei, and their laboratories for their help in experimental approaches and comments on this manuscript. We also thank Dr. Andrey Shaw and Dr. Susan Taylor for their assistances. This study is supported by NCCRF startup grant (NCCRF-SUG-JH), NCCRF bridging grant (NCCRF-YR2016-JULBG1), NMRC seeding grants (NCCSPG-YR2015-JUL-14 and NCCSPG-YR2016-JAN-17), Duke-NUS Khoo Bridge Funding Award (Duke-NUS-KBrFA/2017/0003), Asia Fund Cancer Research (AFCR2017/2019-JH) and SHF Research Grant (SHF/FG692S/2016). The authors declare no potential conflicts of interest.

\section{Reference}

1. A. S. Dhillon, S. Hagan, O. Rath, W. Kolch, MAP kinase signalling pathways in cancer. Oncogene 26, 3279-3290 (2007).

2. J. A. McCubrey, L. S. Steelman, W. H. Chappell, S. L. Abrams, E. W. Wong, F. Chang, B. Lehmann, D. M. Terrian, M. Milella, A. Tafuri, F. Stivala, M. Libra, J. Basecke, C. Evangelisti, A. M. Martelli, R. A. 
Franklin, Roles of the Raf/MEK/ERK pathway in cell growth, malignant transformation and drug resistance. Biochim Biophys Acta 1773, 1263-1284 (2007).

3. P. J. Roberts, C. J. Der, Targeting the Raf-MEK-ERK mitogen-activated protein kinase cascade for the treatment of cancer. Oncogene 26, 3291-3310 (2007).

4. N. A. Brown, L. V. Furtado, B. L. Betz, M. J. Kiel, H. C. Weigelin, M. S. Lim, K. S. Elenitoba-Johnson, High prevalence of somatic MAP2K1 mutations in BRAF V600E-negative Langerhans cell histiocytosis. Blood 124, 1655-1658 (2014).

5. R. Chakraborty, O. A. Hampton, X. Shen, S. J. Simko, A. Shih, H. Abhyankar, K. P. Lim, K. R. Covington, L. Trevino, N. Dewal, D. M. Muzny, H. Doddapaneni, J. Hu, L. Wang, P. J. Lupo, M. J. Hicks, D. L. Bonilla, K. C. Dwyer, M.-L. L. Berres, P. I. Poulikakos, M. Merad, K. L. McClain, D. A. Wheeler, C. E. Allen, D. W. Parsons, Mutually exclusive recurrent somatic mutations in MAP2K1 and BRAF support a central role for ERK activation in LCH pathogenesis. Blood 124, 3007-3015 (2014).

6. E. L. Diamond, B. H. Durham, J. Haroche, Z. Yao, J. Ma, S. A. Parikh, Z. Wang, J. Choi, E. Kim, F. CohenAubart, S. C. Lee, Y. Gao, J.-B. B. Micol, P. Campbell, M. P. Walsh, B. Sylvester, I. Dolgalev, O. Aminova, A. Heguy, P. Zappile, J. Nakitandwe, C. Ganzel, J. D. Dalton, D. W. Ellison, J. Estrada-Veras, M. Lacouture, W. A. Gahl, P. J. Stephens, V. A. Miller, J. S. Ross, S. M. Ali, S. R. Briggs, O. Fasan, J. Block, S. Héritier, J. Donadieu, D. B. Solit, D. M. Hyman, J. Baselga, F. Janku, B. S. Taylor, C. Y. Park, Z. Amoura, A. Dogan, J.-F. F. Emile, N. Rosen, T. A. Gruber, O. Abdel-Wahab, Diverse and Targetable Kinase Alterations Drive Histiocytic Neoplasms. Cancer Discov 6, 154-165 (2016).

7. J. J. Waterfall, E. Arons, R. L. Walker, M. Pineda, L. Roth, J. K. Killian, O. D. Abaan, S. R. Davis, R. J. Kreitman, P. S. Meltzer, High prevalence of MAP2K1 mutations in variant and IGHV4-34-expressing hairy-cell leukemias. Nat Genet 46, 8-10 (2014).

8. R. Roskoski, Jr., MEK1/2 dual-specificity protein kinases: structure and regulation. Biochem Biophys Res Commun 417, 5-10 (2012).

9. Y. D. Shaul, R. Seger, The MEK/ERK cascade: from signaling specificity to diverse functions. Biochim Biophys Acta 1773, 1213-1226 (2007).

10. P. T. Wan, M. J. Garnett, S. M. Roe, S. Lee, D. Niculescu-Duvaz, V. M. Good, C. M. Jones, C. J. Marshall, C. J. Springer, D. Barford, R. Marais, C. Project, Mechanism of activation of the RAF-ERK signaling pathway by oncogenic mutations of B-RAF. Cell 116, 855-867 (2004).

11. G. Hatzivassiliou, J. R. Haling, H. Chen, K. Song, S. Price, R. Heald, J. F. Hewitt, M. Zak, A. Peck, C. Orr, M. Merchant, K. P. Hoeflich, J. Chan, S.-M. M. Luoh, D. J. Anderson, M. J. Ludlam, C. Wiesmann, M. Ultsch, L. S. Friedman, S. Malek, M. Belvin, Mechanism of MEK inhibition determines efficacy in mutant KRAS- versus BRAF-driven cancers. Nature 501, 232-236 (2013).

12. P. Lito, A. Saborowski, J. Yue, M. Solomon, E. Joseph, S. Gadal, M. Saborowski, E. Kastenhuber, C. Fellmann, K. Ohara, K. Morikami, T. Miura, C. Lukacs, N. Ishii, S. Lowe, N. Rosen, Disruption of CRAFMediated MEK Activation Is Required for Effective MEK Inhibition in KRAS Mutant Tumors. Cancer Cell 25, 697-710 (2014).

13. S. J. Mansour, J. M. Candia, J. E. Matsuura, M. C. Manning, N. G. Ahn, Interdependent domains controlling the enzymatic activity of mitogen-activated protein kinase kinase 1. Biochemistry $\mathbf{3 5}$, 15529-15536 (1996).

14. C. Odendall, N. Rolhion, A. Forster, J. Poh, D. J. Lamont, M. Liu, P. S. Freemont, A. D. Catling, D. W. Holden, The Salmonella kinase SteC targets the MAP kinase MEK to regulate the host actin cytoskeleton. Cell Host Microbe 12, 657-668 (2012).

15. E. R. Park, S. T. Eblen, A. D. Catling, MEK1 activation by PAK: a novel mechanism. Cell Signal 19, $1488-$ 1496 (2007).

16. B. Cseh, E. Doma, M. Baccarini, "RAF" neighborhood: Protein-protein interaction in the Raf/Mek/Erk pathway. FEBS Lett 588, 2398-2406 (2014).

17. E. Desideri, A. L. Cavallo, M. Baccarini, Alike but Different: RAF Paralogs and Their Signaling Outputs. Cell 161, 967-970 (2015).

18. W. Kolch, Meaningful relationships: the regulation of the Ras/Raf/MEK/ERK pathway by protein interactions. Biochem J 351, 289 (2000).

19. J. R. Haling, J. Sudhamsu, I. Yen, S. Sideris, W. Sandoval, W. Phung, B. J. Bravo, A. M. Giannetti, A. Peck, A. Masselot, T. Morales, D. Smith, B. J. Brandhuber, S. G. Hymowitz, S. Malek, Structure of the BRAF-MEK Complex Reveals a Kinase Activity Independent Role for BRAF in MAPK Signaling. Cancer Cell 26, 402-413 (2014). 
20. A. J. Bardwell, L. J. Flatauer, K. Matsukuma, J. Thorner, L. Bardwell, A conserved docking site in MEKs mediates high-affinity binding to MAP kinases and cooperates with a scaffold protein to enhance signal transmission. J Biol Chem 276, 10374-10386 (2001).

21. J. F. Ohren, H. Chen, A. Pavlovsky, C. Whitehead, E. Zhang, P. Kuffa, C. Yan, P. McConnell, C. Spessard, C. Banotai, T. W. Mueller, A. Delaney, C. Omer, J. Sebolt-Leopold, D. T. Dudley, I. K. Leung, C. Flamme, J. Warmus, M. Kaufman, S. Barrett, H. Tecle, C. A. Hasemann, Structures of human MAP kinase kinase 1 (MEK1) and MEK2 describe novel noncompetitive kinase inhibition. Nature Structural \& Molecular Biology 11, 1192-1197 (2004).

22. F. Catalanotti, G. Reyes, V. Jesenberger, G. Galabova-Kovacs, R. de Simoes, O. Carugo, M. Baccarini, A Mek1-Mek2 heterodimer determines the strength and duration of the Erk signal. Nat Struct Mol Biol 16, 294-303 (2009).

23. T. O. Fischmann, C. K. Smith, T. W. Mayhood, J. E. Myers, P. Reichert, A. Mannarino, D. Carr, H. Zhu, J. Wong, R.-S. S. Yang, H. V. Le, V. S. Madison, Crystal structures of MEK1 binary and ternary complexes with nucleotides and inhibitors. Biochemistry 48, 2661-2674 (2009).

24. C. J. Caunt, M. J. Sale, P. D. Smith, S. J. Cook, MEK1 and MEK2 inhibitors and cancer therapy: the long and winding road. Nat Rev Cancer 15, 577-592 (2015).

25. S.-H. H. Chen, Y. Zhang, R. D. Van Horn, T. Yin, S. Buchanan, V. Yadav, I. Mochalkin, S. S. Wong, Y. G. Yue, L. Huber, I. Conti, J. R. Henry, J. J. Starling, G. D. Plowman, S.-B. B. Peng, Oncogenic BRAF Deletions That Function as Homodimers and Are Sensitive to Inhibition by RAF Dimer Inhibitor LY3009120. Cancer Discov 6, 300-315 (2016).

26. S. A. Foster, D. M. Whalen, A. Özen, M. J. Wongchenko, J. Yin, I. Yen, G. Schaefer, J. D. Mayfield, J. Chmielecki, P. J. Stephens, L. A. Albacker, Y. Yan, K. Song, G. Hatzivassiliou, C. Eigenbrot, C. Yu, A. S. Shaw, G. Manning, N. J. Skelton, S. G. Hymowitz, S. Malek, Activation Mechanism of Oncogenic Deletion Mutations in BRAF, EGFR, and HER2. Cancer Cell 29, 477-493 (2016).

27. J. Hu, L. G. Ahuja, H. S. Meharena, N. Kannan, A. P. Kornev, S. S. Taylor, A. S. Shaw, Kinase regulation by hydrophobic spine assembly in cancer. Mol Cell Biol 35, 264-276 (2015).

28. J. Hu, E. C. Stites, H. Yu, E. A. Germino, H. S. Meharena, P. Stork, A. P. Kornev, S. S. Taylor, A. S. Shaw, Allosteric Activation of Functionally Asymmetric RAF Kinase Dimers. Cell 154, 1036-1046 (2013).

29. J. Hu, H. Yu, A. P. Kornev, J. Zhao, E. L. Filbert, S. S. Taylor, A. S. Shaw, Mutation that blocks ATP binding creates a pseudokinase stabilizing the scaffolding function of kinase suppressor of Ras, CRAF and BRAF. Proc Natl Acad Sci U S A 108, 6067-6072 (2011).

30. A. P. Kornev, N. M. Haste, S. S. Taylor, L. F. Eyck, Surface comparison of active and inactive protein kinases identifies a conserved activation mechanism. Proc Natl Acad Sci U S A 103, 17783-17788 (2006).

31. A. S. Shaw, A. P. Kornev, J. Hu, L. G. Ahuja, S. S. Taylor, Kinases and pseudokinases: lessons from RAF. Mol Cell Biol 34, 1538-1546 (2014).

32. S. S. Taylor, A. P. Kornev, Protein kinases: evolution of dynamic regulatory proteins. Trends Biochem Sci 36, 65-77 (2011).

33. S. S. Taylor, A. Shaw, J. Hu, H. S. Meharena, A. Kornev, Pseudokinases from a structural perspective. Biochem Soc Trans 41, 981-986 (2013).

34. R. N. Grisham, B. E. Sylvester, H. Won, G. McDermott, D. DeLair, R. Ramirez, Z. Yao, R. Shen, F. Dao, F. Bogomolniy, V. Makker, E. Sala, T. E. Soumerai, D. M. Hyman, N. D. Socci, A. Viale, D. M. Gershenson, J. Farley, D. A. Levine, N. Rosen, M. F. Berger, D. R. Spriggs, C. A. Aghajanian, D. B. Solit, G. Iyer, Extreme Outlier Analysis Identifies Occult Mitogen-Activated Protein Kinase Pathway Mutations in Patients With Low-Grade Serous Ovarian Cancer. J Clin Oncol 33, 4099-4105 (2015).

35. D. S. Nelson, A. van Halteren, W. T. Quispel, C. van den Bos, J. V. Bovee, B. Patel, G. Badalian-Very, P. van Hummelen, M. Ducar, L. Lin, L. E. MacConaill, R. M. Egeler, B. J. Rollins, MAP2K1 and MAP3K1 mutations in Langerhans cell histiocytosis. Genes Chromosomes Cancer 54, 361-368 (2015).

36. G. Galabova-Kovacs, D. Matzen, D. Piazzolla, K. Meissl, T. Plyushch, A. P. Chen, A. Silva, M. Baccarini, Essential role of B-Raf in ERK activation during extraembryonic development. Proc Natl Acad Sci U S A 103, $1325-1330$ (2006).

37. J. Hu, P. Strauch, A. Rubtsov, E. E. Donovan, R. Pelanda, R. M. Torres, Lsc activity is controlled by oligomerization and regulates integrin adhesion. Mol Immunol 45, 1825-1836 (2008).

38. X. Wang, S. E. Boyken, J. Hu, X. Xu, R. P. Rimer, M. A. Shea, A. S. Shaw, A. H. Andreotti, Y. H. Huang, Calmodulin and $\mathrm{PI}(3,4,5) \mathrm{P}(3)$ cooperatively bind to the Itk pleckstrin homology domain to promote efficient calcium signaling and IL-17A production. Sci Signal 7, ra74 (2014). 


\section{Figure Legends}

Figure 1 The $\beta 3-\alpha C$ loop deletion defines a novel group of MEK mutations in cancer genomes. (A) Cancer-related MEK1 mutation spectrum. Cancer-related MEK1 mutations have four hotspots: (I) the inhibitory helix A, (II) the $\alpha \mathrm{C}-\beta 4$ loop, (III) the $\beta 7-\beta 8$ loop, and (IV) the $\beta 3-\alpha C$ loop.

(B) Schematic diagram showing the mutation hotspots of MEK1. The frequently mutated-residues in helix A are colored with blue, and those in hotspot II and III are colored with red. All of these residues in hotspot I, II, and III involve in the interaction of helix A with the kinase domain. The hotspot IV is colored with cyan.

(C) Alignment of MEK1 mutants with $\beta 3-\alpha C$ loop deletions.

(D-E) MEK1 mutants with $\beta 3-\alpha C$ loop deletions are constitutively active. D, MEK1 mutants activate ERK signaling when expressed in 293T cells. E, MEK1 mutants phosphorylate ERK in vitro upon purification from 293T transfectants.

(F) MEK1 mutants with $\beta 3-\alpha C$ loop deletions transform immortalized MEFs. The stable fibroblast cell lines expressing MEK1 mutants were constructed and the foci formation assay was carried out as described before $(27,29)$.

\section{Figure 2 MEK1 mutants with $\beta 3-\alpha C$ loop deletions exhibit a differential inhibitor resistance in} vitro and in vivo. expressed in fibroblasts. Fibroblasts stably expressing different MEK1 mutants were treated with MEK inhibitors as indicated, and phospho-ERK1/2 was probed by immunoblot and quantified by using Image J.

(B-D) Fibroblastomas induced by MEK1 mutants with $\beta 3-\alpha C$ loop deletions have different response to MEK inhibitor treatment. B-C, Fibroblasts expressing different MEK1 mutants were subcutaneously injected NOD-SCID mice which will be treated with or without tramentinib as described in Materials and Methods, and xenograft tumors were harvested and weight. D, Immunohistochemistry staining analysis of xenograft tumors from B. ERK $1 / 2$ activity and cell proliferation were indicated respectively as phosphor-ERK1/2 and Ki67 stainings.

\section{Figure 3 Molecular mechanism that underlies the activation of MEK1 by variable $\beta 3-\alpha C$ loop deletions.}
(A) The $\beta 3-\alpha C$ loop deletions promote the homodimerization of MEK1. MEK1 mutants were expressed in 293T cells and their oligomerization were detected by PAGE with low SDS $(0.1 \%)$. (B) The compound mutation (GGAA) that disrupts the dimer interface blocks the activity of MEK1 mutants with $\beta 3-\alpha C$ loop deletions. MEK1 mutants were expressed in 293T cells, and their activity was measured by phospho-ERK $1 / 2$ immunoblot.

(C) Kinase-dead MEK1 is able to function as allosteric activator to stimulate wild-type MEK1. C-spine fused MEK1 mutants were expressed respectively with WT MEK1 in 293T cells, phospho-ERK1/2 was measured by immunoblot. 
491 (D-F) The activation of MEK driven by homodimerization requires an intra-dimer cross-

492 phosphorylation of AL between two protomers. D, MEK1 $(\Delta \mathrm{EI})$ and MEK1 $(\Delta \mathrm{HLEI})$ auto-phosphorylate

493 their AL, which is blocked C-spine fusion. MEK1 mutants were purified from 293T transfectants and

494 the phospho-AL was detected by immunoblot. E, the AL of MEK1 receiver is not phosphorylated when

495 coexpressed with allosteric activators. The wild-type MEK1 was co-expressed with kinase-dead

496 allosteric MEK1 mutants, and its AL-phosphorylation was detected by immunoblot upon

497 immunoprecipitation. F, The non-phosphorylatable AL-mutation decreases the activity of MEK1 $(\triangle \mathrm{EI})$

498 and MEK1( $\triangle \mathrm{HLEI})$. MEK1 mutants were expressed in 293T cells, and phospho-ERK1/2 in

499 transfectants was detected by immunoblot.

500 (G) A model of homodimerization-driven MEK activation. Homodimerization helps MEK assembling an

501 active conformation, which in turn induces a cross-phosphorylation of AL between two protomers. The

502 AL-phosphorylation fully activates MEK dimer and stabilizes its active conformation. Then MEK dimer

503 with phospho-AL catalyzes the phosphorylation of ERK AL.

504

505 Figure 4. The dimerization of MEK is critical for its phosphorylation by active RAF and for its 506 catalytic activity towards ERK.

507 (A-B) The phosphorylation of MEK by active RAF requires its dimerization. A, Wild-type MEK1 and the 508 dimer-enhanced MEK1 mutant but not the dimer-impaired MEK1 mutant are phosphorylated by active 509 RAF. MEK1 mutants were co-expressed with either BRAF(V600E) or K-ras(G12V) in 293T cells, and

510 their AL-phosphorylation is detected by immunoblot upon immunoprecipitation. B, Both dimer-

511 enhanced and dimer-impaired MEK mutants barely bind to active RAF. MEK1 mutants were co-

512 expressed respectively with BRAF(V600E) and their dimerization was detected by co-

513 immunoprecipitation. MEK1(M308/310A) that has an impaired ability to dimerize with RAF kinase

514 serviced as a control.

515 (C) Active MEK functions as a dimer to activate ERK. The disruption of dimer interface inhibits the

516 activity of MEK1(DD), which can be rescued by GST fusion. In contrast, this does not occur in

$517 \operatorname{MEK} 1(\Delta \mathrm{EI})$, since it is triggered by enhanced-dimerization. MEK1 mutants were expressed in 293T

518 cells, and their activity was measured by phospho-ERK1/2 immunoblot.

519 (D) Both wild-type and dimer-impaired ERK can be phosphorylated by MEK. ERK2 mutants were

520 expressed with either MEK1(DD) or BRAF(V600E) in 293T cells, purified by immunoprecipitation, and

521 their AL-phosphorylation was detected by immunoblot.

522 (E) A model for the activation of RAF/MEK/ERK kinase cascade. I. In quiescent cells, RAF/MEK forms

523 a face-to-face heterodimer in cytosol. II. Upon stimulation, RAF/MEK heterodimers are recruited to the

524 plasma membrane by active RAS through the RBD domain of RAF, where they form a transient

525 tetramer through the side-to-side RAF dimerization. III. The side-to-side RAF dimerization not only

526 turns on RAF but also loosens its face-to-face heterodimerization with MEK, and therefore facilitates

527 the homodimerization of MEK on the surface of RAF dimer. IV. Active RAF dimer activates the MEK

528 dimer docking on its surface by phosphorylating its $A L$, and MEK dimer can also activate itself through

529 homodimerization-driven AL-auto-phosphorylation. V. Active MEK dimer docking on or releasing from

530 the surface of RAF dimer phosphorylates ERK. 


\section{Figure 1}

A
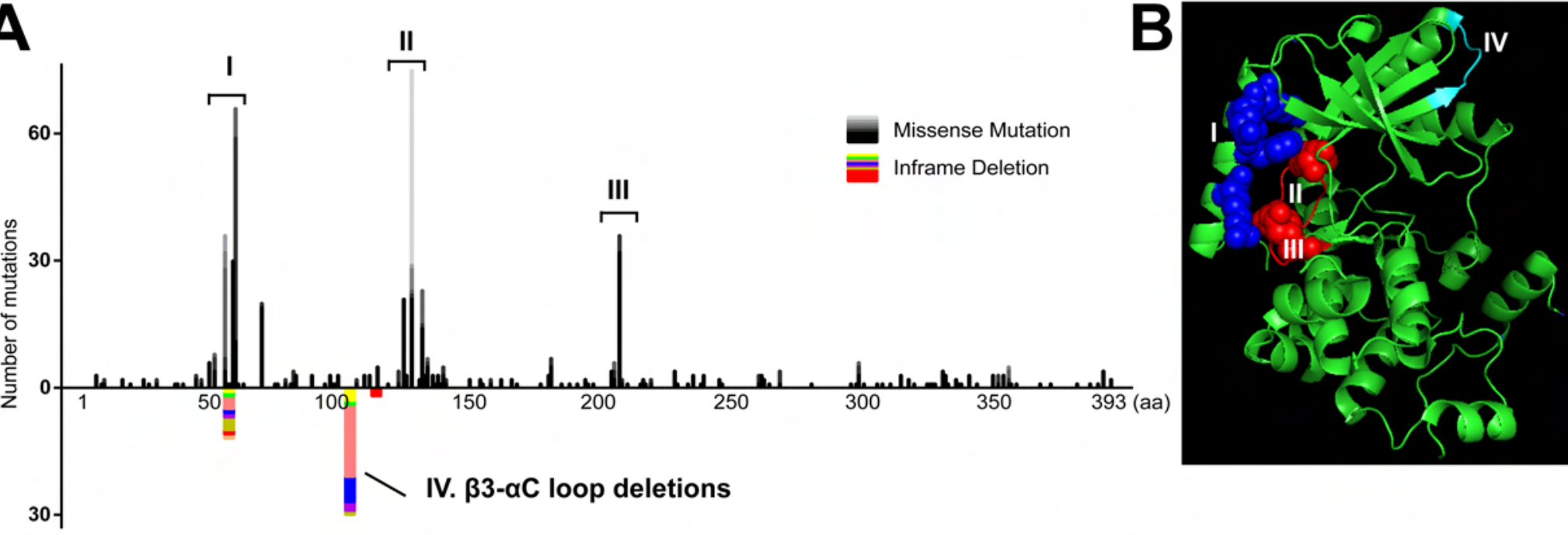

C

\section{$\beta 3$}

$\alpha \mathrm{C}$

MEK1
MARKLIHLEIKPAIRNQI IRELQVLHECN MARKL------PAIRNQI IRELQVLHECN MARKLIPL--KPAIRNQI IRELQVLHECN MARKLIHL--KPAIRNQI IRELQVLHECN MARKLIHLE--PAIRNQI IRELQVLHECN MARKLIHLEIK--IRNQI IRELQVLHECN MARKLIHLEIKL--RNQI IRELQVLHECN MARKLIHLEIKPAIR-----ELQVLHECN
No.

D

흥

플

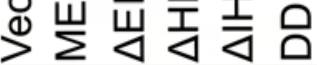

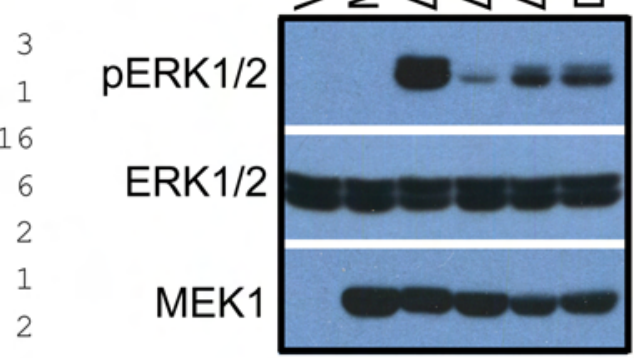

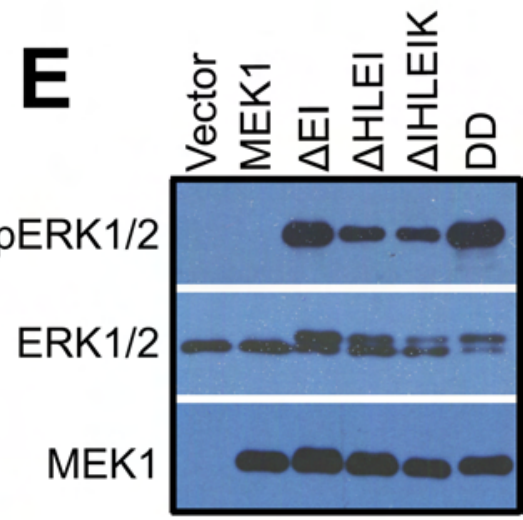

E
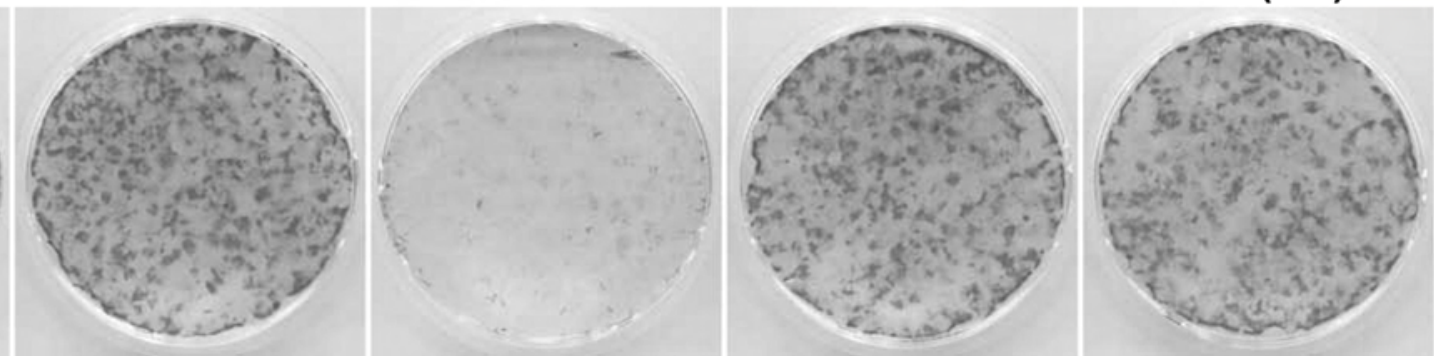


\section{Figure 2}

\section{A}
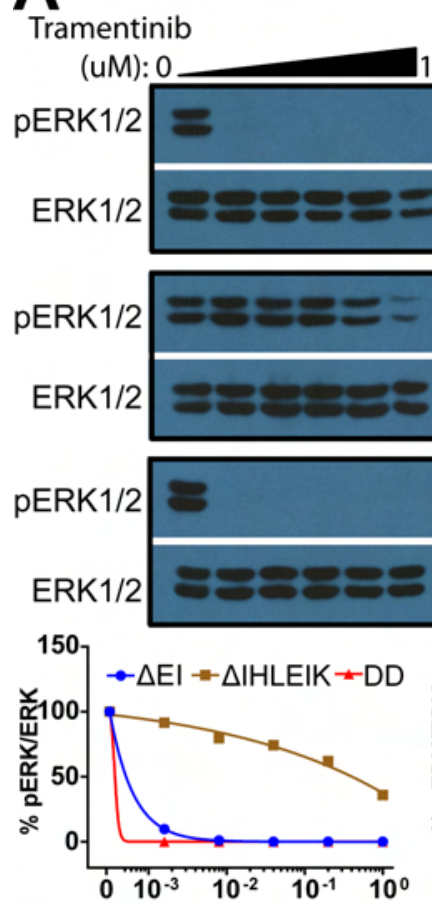

B

\section{Gen Drug}

MEK1 -

$\Delta \mathrm{EI}$

$\Delta \mathrm{EI}+$

$\triangle$ IHLEIK - M

$\triangle \mathrm{IHLEIK}+$

DD - $0|x|$ क

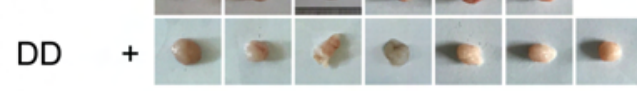

D

Trametinib: $\quad-\quad+$

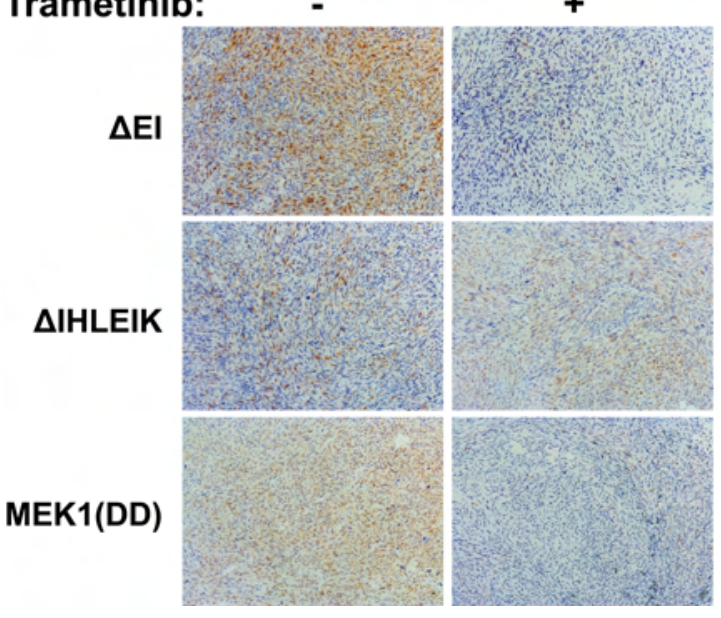

Cobimetinib

GDC0623
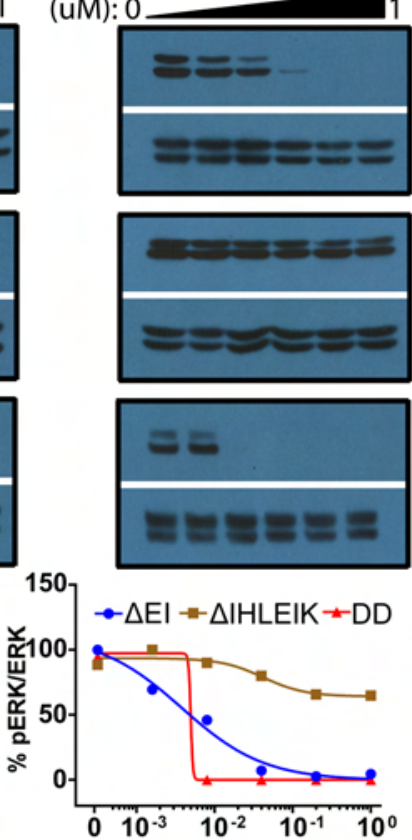

(uM): 0
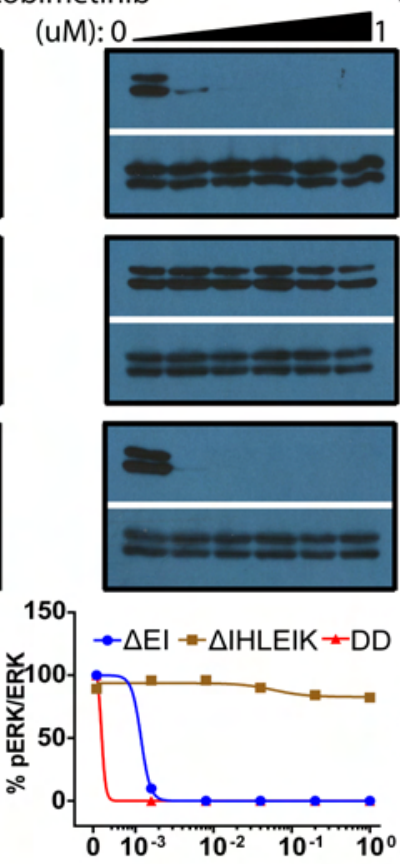

AZD6244

(uM): 0
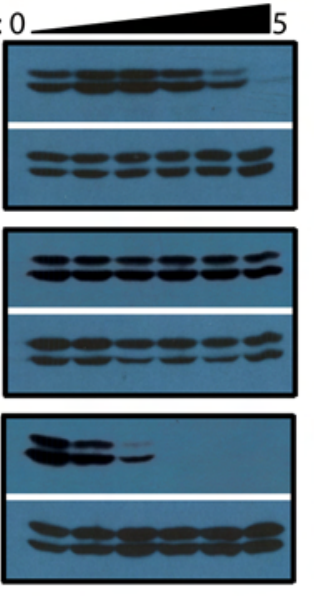

$150, \triangle E I-\triangle H H E E I K \rightarrow D D$

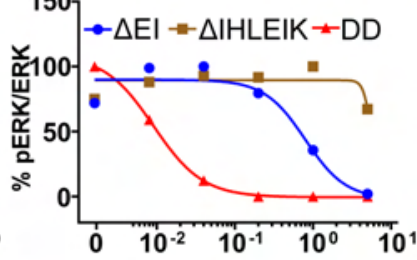

Binimentinib
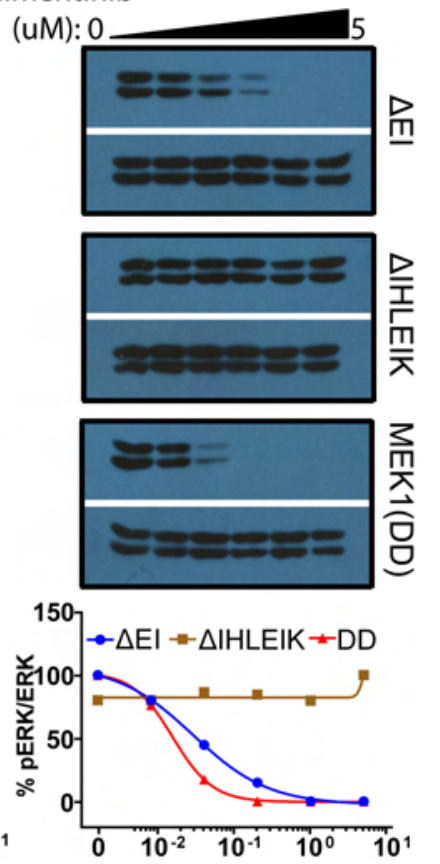

\section{C}
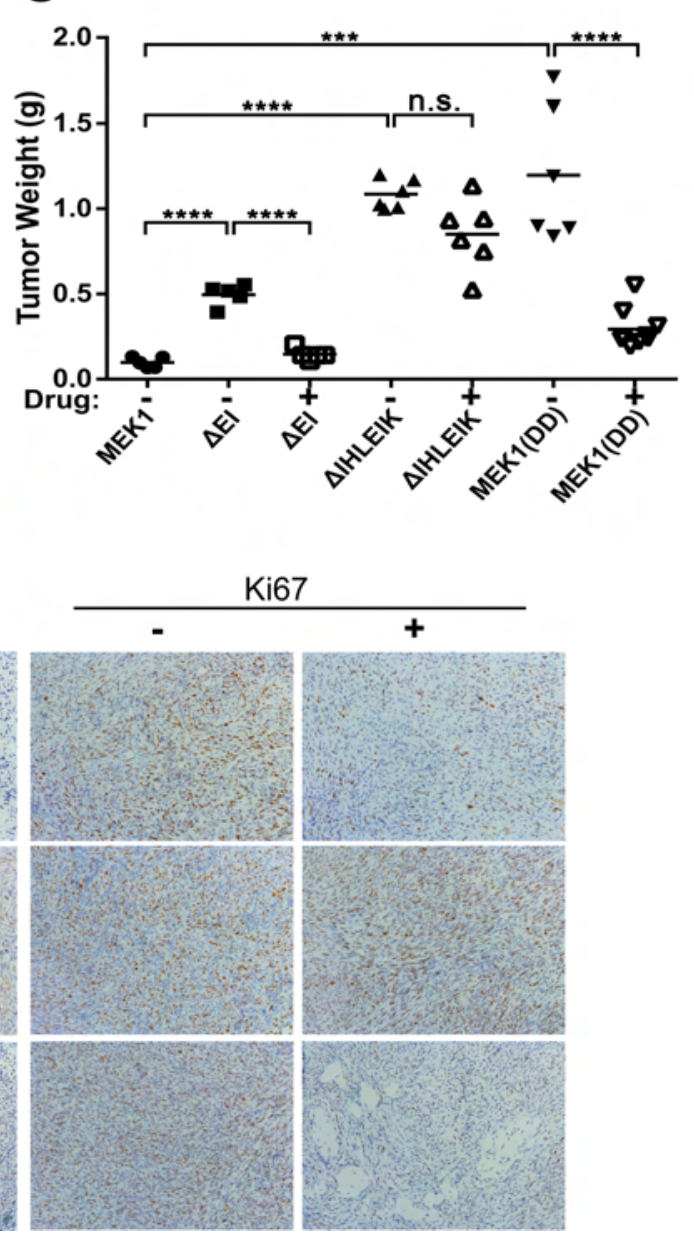
Figure 3

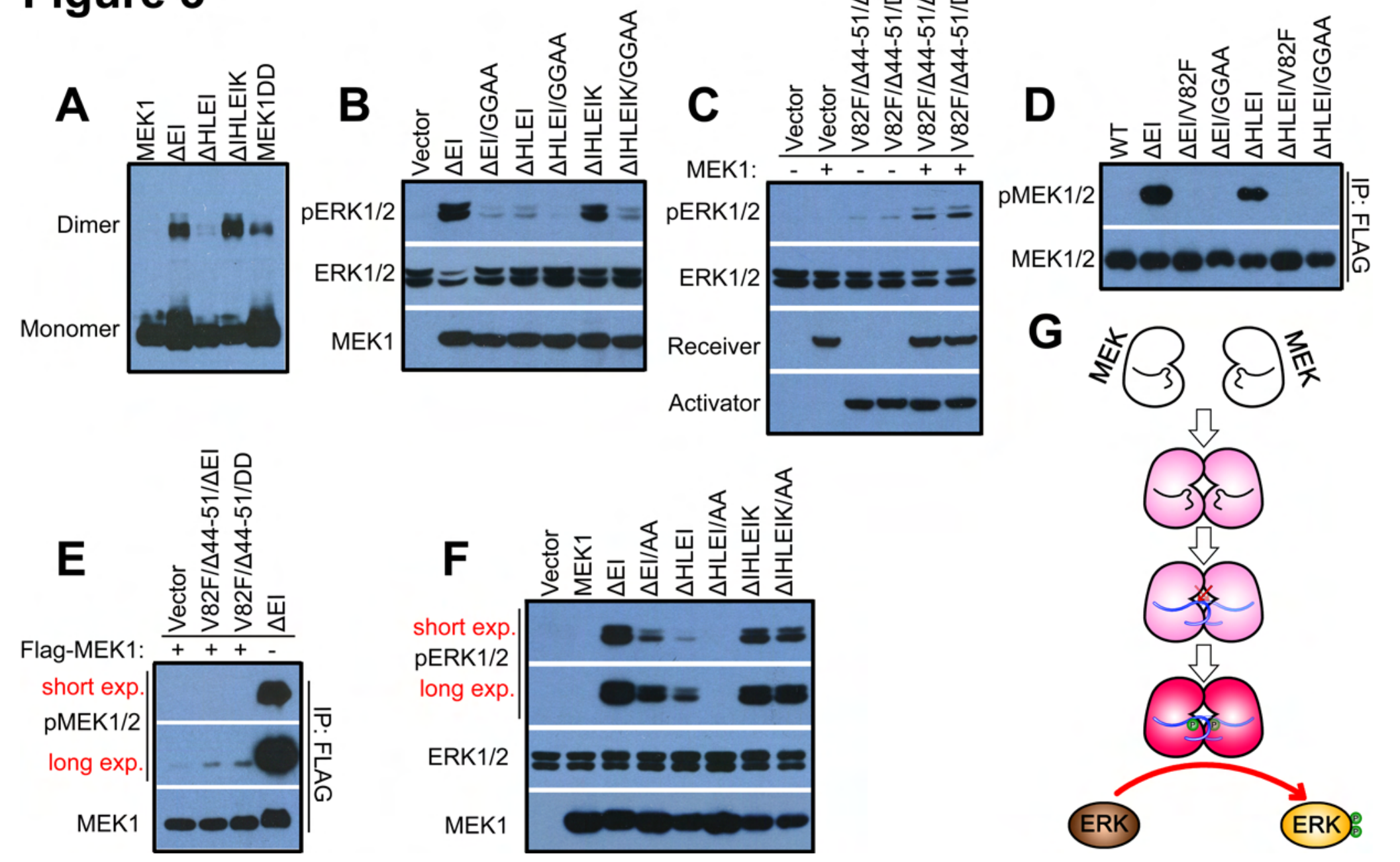




\section{Figure 4}

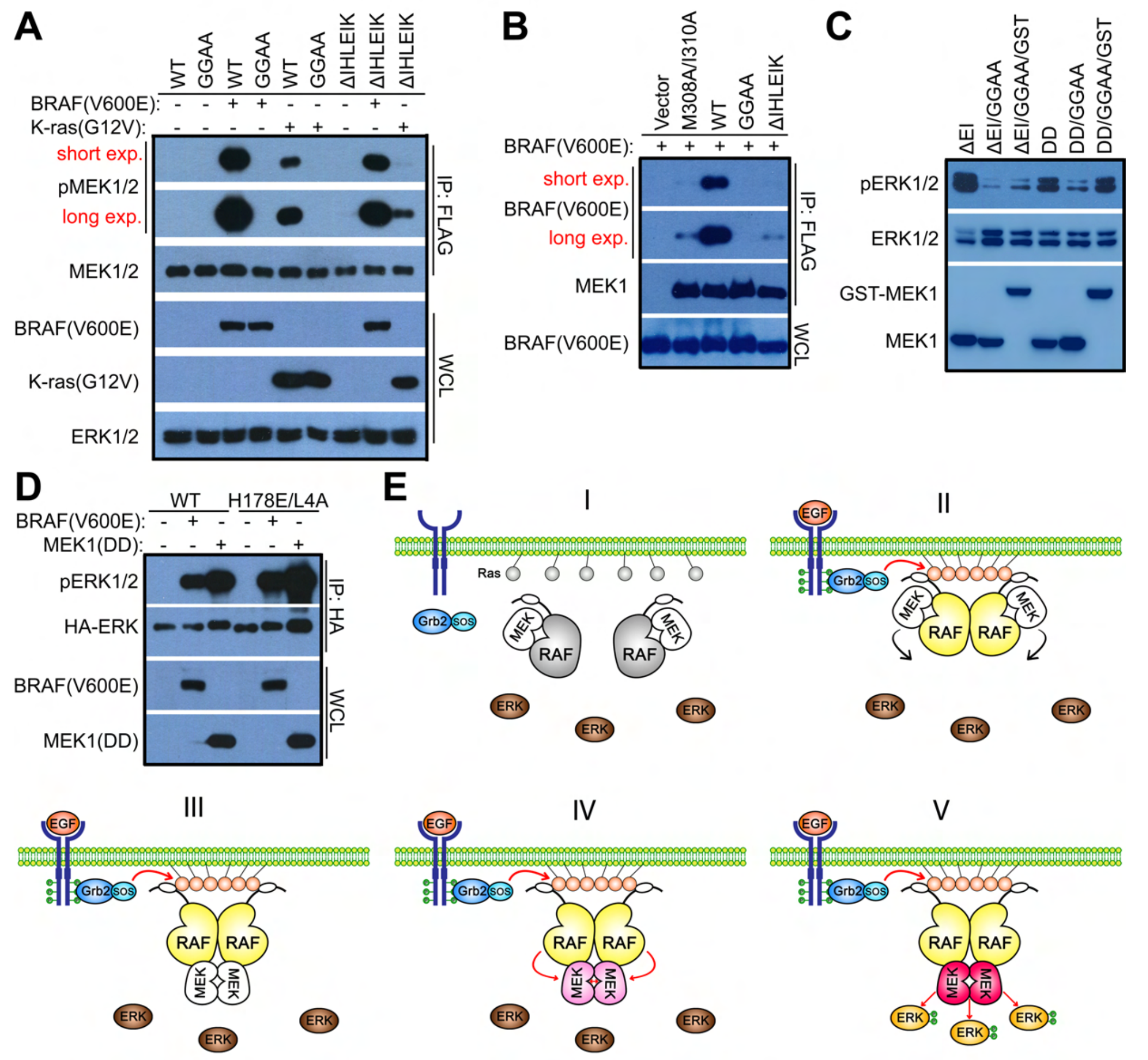

